\title{
Impact of Energy Storage on Cascade Mitigation in Multi-energy Systems
}

\author{
Mads Almassalkhi, Student Member, IEEE
}

\author{
Ian Hiskens, Fellow, IEEE
}

\begin{abstract}
In this paper, we establish energy-hub networks as multi-energy systems and present a relevant model-predictive cascade mitigation control (MPC) scheme within the framework of energy hubs. The performance of both open- and closed-loop mitigation schemes is investigated for various energy storage scenarios. The results are illustrated using a small 11-hub network and a larger 69-hub network and show that sizing and performance ratings of energy storage devices have significant effect on cascade mitigation control in multi-energy systems. Specifically, we conclude that increasing energy storage capacity and limiting the rate of energy delivery improves long-term performance of our closed-loop MPC scheme.
\end{abstract}

Index Terms-Energy hub, model-predictive control, reliability, energy storage, multi-energy system, random networks.

\section{INTRODUCTION}

$\mathbf{R}$ ECENTLY, large-scale power grid failures have placed a renewed focus on the reliability and optimality of energy supply systems [1]. Such systems extend beyond electrical power to include various energy carriers, such as natural gas, hydro, and wind energy. In fact, coupling energy carriers may reveal minimum cost solutions that are not apparent when each energy system is treated separately [2]. By employing energy hubs we move beyond the traditional view and consider largescale coupled energy systems (multi-energy systems).

An energy hub represents a relatively new and general concept in power systems, where the couplings between multiple energy networks are explicitly modeled in the form of conversion processes [2], [3]. In addition, hub models seamlessly allow for the inclusion of energy storage. Our previous work [4] developed a model predictive cascade mitigation scheme for large-scale energy-hub systems, which shed minimal load in the process of halting the cascade. The effectiveness of the cascade mitigation process in maximizing economic and secure operation was due to proper management of available energy storage. Therefore, in this paper, we are specifically interested in the effects of different energy storage scenarios on the cascade mitigation scheme. The scenarios will reflect energy storage under various power and energy rating assumptions.

Energy storage has been studied extensively in decoupled electrical networks as a means of reducing the effect of intermittent sources [5], [6], [7]. Furthermore, cascading failures in multi-energy systems have been previously studied outside of the scope of energy hubs, but such studies, generally, do not consider an active role for energy storage [8], [9].

This work was supported by U.S. Department of Energy under research grant DE-SC0002283.

M. Almassalkhi and I. Hiskens are with Department of Electrical Engineering and Computer Science, University of Michigan, 1301 Beal Avenue, Ann Arbor, MI 48109, U.S.A. \{malmassa, hiskens\}@umich.edu
In [10], the authors investigated expansion planning in multienergy systems, including natural gas underground storage, but focused on minimizing operational costs and did not consider contingency situations.

Employing the optimization framework developed in [11], we construct large energy-hub systems and investigate these systems under multi-line outages. In a general sense, power flows across transmission lines and pipelines are compared against proper flow limits, and when violations occur, the lines are taken out of service. There exists a myriad of approaches to determine when an overloaded line should be removed from service, ranging from deterministic hard constraints with memory, as in [12], to soft-constrained probabilistic schemes described in [13]. Incorporation of line-tripping into our model is accomplished by employing a mixed-integer disjunctive model [14]. To mitigate the effects of a disturbance and prevent cascading failures, we employ our model predictive cascade mitigation controller [4].

Our paper is organized as follows. In Section II, we provide an overview of energy hub concepts. The model predictive cascade mitigation control (MPC) scheme is summarized in Section III. In Section IV, we discuss energy storage under the energy hub paradigm. The MPC scheme is employed in Section $\mathrm{V}$ under various energy storage scenarios for two different energy hub networks. Section VI presents the simulation results, while Section VII concludes the paper with remarks on future work.

\section{MODEL}

Multi-carrier energy networks may be formulated in different ways. This paper will focus the discussion on the "hybrid energy hub" model initially developed by [2]. Under this formulation, we allow the system operator of an energy hub network to directly manipulate and control line-switching, load-shedding and converter, generator, and energy storage utilization. For mathematical details on the models in the following sections, we refer the reader to [11].

\section{A. Energy Hubs}

Most common energy hubs consist of five simple interconnected elements: inputs, input-side energy storage, energy converters, output-side energy storage, and outputs. To properly describe the flow of power through the energy hub, we need to describe how power flows between these elements. Energy carrier networks feed power into the hub at the input side, where storage may be available. The power that was not utilized for storage is dispatched into converters that transform the energy accordingly. On the output side of the 


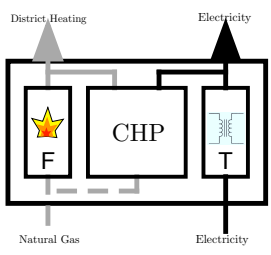

(a) Hub converter example

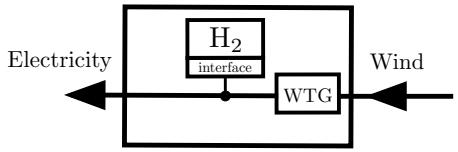

(b) Hub storage example

Fig. 1. Simple and common illustrations of energy hubs

hub, converted power may be utilized for storage or injected into the carrier network connected to that side.

One simple example of an energy hub is shown in Figure 1(a) where a furnace, a transformer, and a combined heat and power (CHP) plant are considered part of the the same energy hub. The inputs to the hub are electrical and natural gas power flows and the outputs are district heating and electrical power flows. The electrical energy is converted to low-voltage electrical energy by the transformer with some efficiency and injected into a low-voltage grid on the output side. The natural gas flowing into the hub can be dispatched to a furnace (for heating) or to a CHP plant where natural gas is converted into both low-voltage electrical energy and district heating.

To illustrate the inclusion of storage devices within the energy hub formulation, consider Figure 1(b), wherein wind energy acts as the hub input. The wind turbine converts incoming wind energy into electrical energy. On the output side, the hub contains energy storage (e.g. electrolysis + hydrogen fuel cell storage), which is utilized by charging and discharging the storage device (at possibly different charging and discharging efficiencies) through a storage interface. The electrical power that was not utilized by the output storage device is then injected into an electrical grid or load.

\section{B. Interconnection of Energy Hubs}

Energy hubs are interconnected via multiple energy supply networks. The previous section defined how power flows through an energy hub, however, to describe the flow between energy hubs, we need to consider power networks. A power network is a collection of a set of nodes (e.g. buses) and a set of arcs (e.g. transmission lines) that define a simple graph, as shown in Figure 2. The nodes either consume power from the network (i.e. loads), inject power into the network (i.e. generators), or act as throughput nodes that neither inject nor consume power. If we neglect power losses, we know that the sum of power flows into the network must equal the sum of flows out of the network. In addition, we need to consider the power flows related to energy hub inputs and outputs as consumers and injectors, respectively. Note that the couplings between energy hubs and power networks take place exclusively at the hub inputs and outputs.

Besides being connected to energy hubs, the main difference between a simple graph and a power network lies with the physics of the particular power flows. That is, there exists a physical relationship between the flow across an arc and the connected nodes. For a simple example, consider an electrical

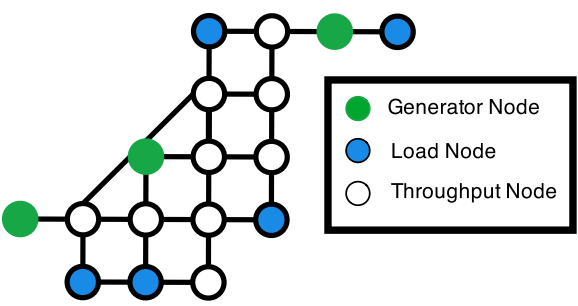

Fig. 2. Representation of a power network as a graph

power network and the linear DC flow model:

$$
x_{i j} f_{i j}=\left(\theta_{i}-\theta_{j}\right)
$$

where $x_{i j}$ represents the reactance of arc $(i, j), f_{i j}$ is the power flow across said arc, and $\theta_{i}$ represents the phase angle of node $i$. In (1), we see that the electrical power flowing across any arc depends on the difference in the phase angle between connected nodes and the reactance of the arc. This physical relationship between nodes and arcs manifests itself differently depending on the nature of the power network and may be linear (e.g. DC model) or non-linear (e.g. AC model).

In this paper, for simplicity, we assume no losses in power networks. Furthermore, since the focus is on interactions between energy carriers, we employ the DC flow model in electrical networks and linearize the natural gas power flows about nominal parameter values to maintain linearity in the representation of the energy hub networks.

\section{Line-outages}

The power flowing across an arc should not exceed the recommended flow limits as it may permanently damage the arc. Therefore, sensors are often in place to allow protective processes to automatically take arcs out of service when the flow exceeds the limit. For example, electrical transmission lines have power flow limits that prevent dangerous sagging and permanent damage. They are related to the thermal capacity of the conductor and the current flowing through the line. Generally, there is an inverse relationship between the overload on a line and the time allowed before the line must be taken out of service and, in most common overload scenarios, the timeresponse is of the order of minutes. While implementation of realistic line-outage models is currently a subject of ongoing research, for this paper, we assume that lines can withstand an overload for 5 minutes before being taken out of service. Removing an arc from service is commonly referred to as "tripping" the arc.

To allow the system controller to trip lines, we employ a mixed integer disjunctive model, which ensures that tripped lines are effectively removed from network models and that nodes of the tripped lines do not directly affect one another. For example, we can incorporate line-tripping into the DC flow model by the following mixed-integer formulation:

$$
\begin{aligned}
\left|f_{i j}\right| & \leq s_{i j} u_{i j} \\
\left|f_{i j} x_{i j}-\left(\theta_{i}-\theta_{j}\right)\right| & \leq M\left(1-s_{i j}\right) .
\end{aligned}
$$

Thus, if transmission line $(i, j)$ is in service $\left(s_{i j} \equiv 1\right)$, the disjunctive formulation enforces power flow limits $\left(\left|f_{i j}\right| \leq u_{i j}\right)$ 
and (1), as should be expected. However, if line $(i, j)$ is tripped $\left(s_{i j} \equiv 0\right)$, the formulation ensures that $f_{i j} \equiv 0$ and the relationship between $\theta_{i}$ and $\theta_{j}$ in (1) becomes inactive (i.e. $\left|\theta_{i}-\theta_{j}\right| \leq M$, where $M$ is large).

\section{Economic Dispatch in Energy Hubs}

Under normal conditions, we schedule the energy hub system on a slow timescale, which consists of one-hour timesteps. The system is operated based on an economic dispatch schedule computed off-line from the Multi-Period Optimal Dispatch Formulation (MPODF) described in detail in [11]. Note that this formulation represents the steady-state system model and takes into account transmission flow limits to maintain system integrity and avoid line overloads. In short, the MPODF schedule satisfies forecasted nominal demand and minimizes the cost of generation by optimal utilization of available hub storage and expected externally injected power (for example wind power) from hour 1 to hour 24. The schedule acts as a reference signal and informs the operator of how to utilize generators, hub converters, and hub storage devices, which lines (if any) should be taken out of service, and how much demand can be satisfied over the entire operating period.

At each time-step, the operator then implements the schedule's recommendations on the actual system (real-time). If our model of the system is perfect over the entire period (including forecasts of renewables and demand), then the line flows will always be within their limits and the system is, in fact, operated optimally. However, if a disturbance takes place (e.g. a large storm) that alters the real system (e.g. a multi-line outage), our model no longer reflect reality and the schedule's recommendations may be suboptimal. Furthermore, continued implementation of the schedule, under such conditions, may exacerbate the effects of the disturbance.

\section{Cascade Mitigation Control}

Cascade failures are initiated when a disturbance takes place that forces a redistribution of flows, which causes additional line-overloads and, subsequently, takes more lines out of service. If left uncontrolled, the cycle of line outages and redistribution of flows is referred to as a cascade failure.

There are two general approaches to mitigating cascade failures in power networks. The first method predicts potential disturbances a priori and is based on off-line computation of all possible or likely failures in the network. Control policies are devised to deal with each failure. A major drawback of this approach is that it does not scale well with the size of the network. The second method is based on retroactive control, whereby the uncertainty surrounding the disturbance has been revealed and one can utilize the knowledge available about the disturbance to determine control responses in real-time to mitigate the effects of the disturbance. This paper focuses on the latter approach and considers a model predictive control scheme. The following steps describe the basic notion behind MPC:

1) Determine a control profile that optimizes a cost criterion over a prediction window,
2) Apply this profile until new process measurements become available,

3) When new measurements are available, repeat step (1).

Once a multi-line outage is detected by the system monitor, operation of the system is switched from the hourly MPODF schedule to the fast timescale MPC scheme (one-minute timesteps). During the fast timescale, nominal load and intermittent power injections are fixed at their most recent slow timescale values, and generation and storage energy delivery rates are taken into account. In our model, if lines are still overloaded after five minutes, they automatically trip. Therefore, our MPC prediction window is five minutes and we assume new measurements are available every minute. Furthermore, in attempting to halt the cascade, we only allow load shedding at minute 5, as a last resort to bring line flows within their limits. Accordingly, there is no reason for our prediction window to extend beyond minute 5 and, therefore, needs to shrinks by one minute after every measurement update. This method of MPC is often referred to as "fixed-point" or "shrinkinghorizon" MPC [15].

Thus, when the MPC scheme is initiated by the detected disturbance (at minute 0), MPC considers a 5-minute prediction window and determines the optimal schedule for generation and storage over the 5-minute horizon to minimize loadshedding at minute 5 . The first instance of this schedule is then applied to the system. At minute 1, new measurements are acquired from the system and a 4-minute prediction window is considered to determine a new optimal schedule over the remaining period. The first instance of the new schedule is applied to the system and the process is repeated. This iterative scheme continues until minute 4 , when MPC considers a 1minute prediction window. At that time, MPC determines the optimal actions to take over that one remaining minute, along with the minimal amount of load to shed to ensure line flows are within their limits at that final time.

If the MPC model has no errors and matches the real system, no overloads will remain, and the cascade will be halted. On the other hand, if the MPC model is imperfect, some overloads may remain. If so, those lines will trip, signaling MPC to repeat the entire process. This continues until no further line tripping occurs. At that point, the slow-timescale MPODF is updated with the latest values for generation, storage and load, and the optimal schedule over the remainder of the 24-hour period is determined. Note that without the lookahead feature of MPC, a closed-loop controller acting only at minute 5 would shed more load, as it would not be able to properly allocate storage utilization to overcome possible future generator power limits. An overview of the energy hub system operation under closed-loop control is illustrated in Figure 3. For more details on cascade mitigation, we refer the reader to [4].

In the case of open-loop control, the system operator implements the MPODF schedule until a disturbance occurs. At this time, the operator seeks only to satisfy demand and line flows are given by the power flow solution with no regard for line flow limits. Therefore, the open-loop control will likely undergo cascading failures. 


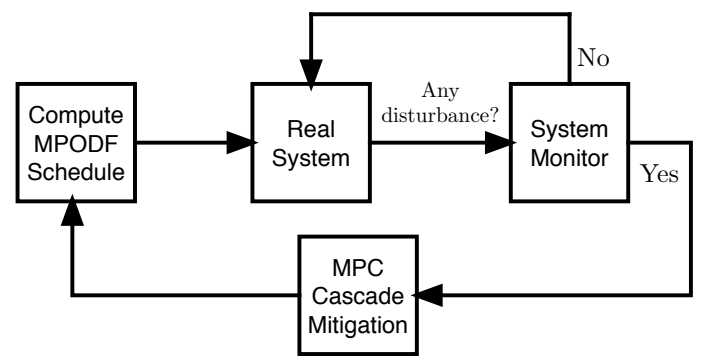

Fig. 3. Operation overview of energy hub system under both normal operating conditions and contingency situations.

\section{ENERGY STORAGE}

During normal operation of the energy hub system, energy storage plays a significant role in minimizing generation costs from conventional generators, as it allows the system operator to pre-position energy in storage during off-peak hours (and pricing) to satisfy demand in the presence of intermittent generation (e.g. wind power). In the previous section, we employed a method to mitigate cascade failures in multienergy systems by determining proper utilization of storage and generation. In fact, hub storage also plays a significant role in cascade mitigation since it acts as a "buffer" against disturbances. That is, a system operator can employ stored energy to satisfy temporary energy shortages or overflows, while allowing time for conventional generators to effectively reconfigure their schedules.

Thus, the effectiveness of system operations in minimizing costs and rejecting disturbances depends on the available energy hub storage infrastructure. Indeed, siting, sizing, and operational capability (e.g. power rating and standing losses) are salient storage parameters. Siting is important for reducing congestion during peak hours; however, the process for determining optimal location of energy hubs is non-trivial and is not considered here. Instead, this paper will fix the location of hubs within the system and study the effects of varying both hub storage capacity and charge/discharge power limits on the cascade mitigation process.

Under the energy hub paradigm, we are able to combine multiple types of energy systems and study their combined performance. Therefore, we need to consider multiple types of energy storage, namely, natural gas storage, electrical storage, and thermal storage. Note that, in this paper, we consider energy storage to have no standing losses, constant charging and discharging efficiencies (see Table I), and we neglect the economics of construction, operation, and maintenance of storage facilities. That is, energy storage represents a cost-free service available to the system operator.

\section{A. Natural Gas Energy Storage}

The two main methods used in industry for storing natural gas are: "packed" pipelines and underground storage facilities. The packing of pipelines refers to the accumulation of natural gas in pipelines. Since such practices are commonly employed for pressure-regulation and only make up a small percentage of natural gas storage capacity, packing of pipelines is not
TABLE I

SUMMARY OF DIFFERENT TYPES OF ENERGY STORAGE

\begin{tabular}{lccc}
\hline Storage & Location & Charge Eff. & Discharge Eff. \\
\hline Natural gas & Only input-side & $99 \%$ & $99 \%$ \\
Hydrogen & Both sides & $80 \%$ & $65 \%$ \\
Thermal & Only output-side & $100 \%$ & $60 \%$ \\
\hline
\end{tabular}

considered in this paper. Instead, we consider underground storage in the form of reshaped salt caverns, which have high throughput and can be cycled hourly for both electric and heating loads, and aquifers, which can be regularly withdrawn and have large capacity [16]. The energy hubs that convert natural gas in the proposed multi-energy systems, therefore, contain input-side storage devices that reflect highly efficient underground storage facilities.

\section{B. Electrical Energy Storage}

With the intermittency of renewable energy (e.g. solar and wind), effective implementation of storage is highly desirable for improving system reliability. While pumped hydro storage and compressed air systems provide two large-scale methods for storing electric-ready power, our focus is mainly on distributed storage in the form of hydrogen storage. Therefore, energy hubs that convert from electrical energy contain inputside hydrogen storage, while hubs that convert to electrical energy (e.g. wind) are outfitted with output-side hydrogen storage (e.g. Figure 1(b)). Hydrogen storage requires an electrolytic process for charging (i.e. create hydrogen) and employs efficient fuel cells during discharging (i.e. consume hydrogen).

\section{Thermal Energy Storage}

Under the energy hub paradigm, both natural gas and electrical energy can be converted into thermal energy to satisfy district heating loads. This inherent energy flexibility improves system reliability and by employing thermal energy storage within the hub, we can satisfy distributed thermal loads from stored thermal energy, which reduces network congestion that tends to arise during peak demand. There exists a wide range of thermal energy storage solutions ranging from molten salt to gas-fired and electric storage heaters. However, for this paper, we will just consider a general form of thermal storage that supplies each heating load. The thermal storage device is employed on the output-side of hubs which convert electrical and natural gas energy into heating. We assume a loss-less conversion of natural gas and electrical energy into thermal storage and attribute thermal energy losses to the discharging process.

Note that the efficiencies in Table I represent optimistic scenarios to give best-case performance of a multi-energy system with storage.

\section{Simulation}

The formulation of cascade mitigation in energy hub networks described in [4] and summarized in Section III, permits the construction of arbitrarily large interconnected energy 
TABLE II

TOPOLOGICAL CHARACTERISTICS OF THE 11-HUB ENERGY NETWORK

\begin{tabular}{lccccc}
\hline Network & $\langle k\rangle$ & $N$ & $E$ & $G$ & $D$ \\
\hline Electrical & 1.67 & 6 & 5 & 1 & 3 \\
Gas & 1.67 & 6 & 5 & 3 & 1 \\
Wind & 0 & 2 & 0 & 2 & 0 \\
Heat & 0 & 4 & 0 & 0 & 4 \\
\hline
\end{tabular}

TABLE III

TOPOLOGICAL CHARACTERISTICS OF THE 69-HUB ENERGY NETWORK

\begin{tabular}{lccccc}
\hline Network & $\langle k\rangle$ & $N$ & $E$ & $G$ & $D$ \\
\hline Electrical & 3.07 & 60 & 92 & 20 & 15 \\
Gas & 3.00 & 60 & 90 & 12 & 21 \\
Wind & 0 & 18 & 0 & 18 & 0 \\
Heat & 0 & 22 & 0 & 0 & 22 \\
\hline
\end{tabular}

hub networks. Together with CPLEX and MATLAB, we can implement our model predictive cascade mitigation scheme outlined in Figure 3.

In this paper, we analyze the performance of the MPC scheme under different energy storage scenarios. That is, we investigate how the availability (i.e. capacity) and performance (i.e. charge/discharge power limits) of energy storage devices impact the amount of load shed.

To achieve this we employ the random grid generating techniques proposed in [17] to construct two multi-energy systems - a small 11-hub system and a larger 69-hub system and subject each to a multi-line outage. The parameters used to construct the power grids are given in [4, Table II]. The small energy hub system is shown in Figure 4. Due to the size of electrical and natural gas networks and the random interconnectivity with energy hubs, a meaningful visualization of the larger energy hub system is not straightforward and is excluded. The smaller simple system allows us to better describe how storage is utilized to mitigate cascade failure; however, the larger system is useful to include in the discussion, as it exhibits meaningful cascade behavior.

The topological characteristics of our systems are given in

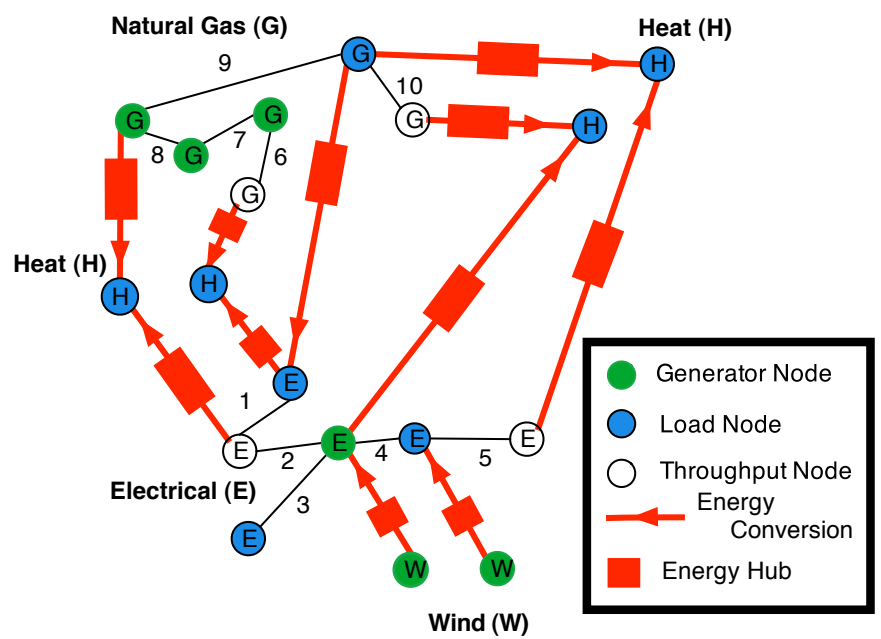

Fig. 4. Network representation of small 11-hub system.
TABLE IV

CONVERSION EFFICIENCIES BETWEEN ENERGY TYPES

\begin{tabular}{lcccc}
\hline From $\backslash$ To & Electric & Gas & Wind & Heat \\
\hline Electric & - & - & - & 0.75 \\
Gas & 0.70 & - & - & 0.90 \\
Wind & 1.00 & - & - & - \\
\hline
\end{tabular}

Tables II and III. The values $N, E, G$, and $D$ represent the number of nodes, arcs, generators, and loads, respectively, while $\langle k\rangle$ is the average nodal degree. The wind and heat networks have no arcs and consist exclusively of generators and loads, respectively. We employed 11 energy hubs in the small system and 69 energy hubs in the large system to couple the four different energy networks. The energy hubs are used to connect from the electrical network to heating loads (via resistor heating), from the gas network to electrical (via gas turbines) and heat networks (via furnaces), and from the wind network to the electrical network (via turbines). All other network couplings, for example from gas to wind and from heat to electric, are excluded in this simulation.

Table IV shows the energy conversion efficiencies employed in our simulation. Note that the efficiency between wind energy and electric energy is set to 1.0 , because we only consider injected power from the wind generators and the lossy conversion between wind speed and electricity production is assumed to have already taken place. All hubs connecting the wind network to the electrical network have output storage, while applicable input and output hub storage is added randomly to all of the remaining hubs.

The system is assigned 24 one-hour time-intervals, corresponding to one complete day of operation. For the fast timescale, network arcs are allowed to be overloaded for no longer than 5 minutes before being tripped automatically. The consumer demand is set to peak near midday, while wind power is available mostly in the early and later parts of the day. The cost of generation (electric and natural gas) is set to vary linearly with forecast demand, so generation near midday is more expensive than during the earlier or later parts of the day. Electric generation is set to be more costly than natural gas. Finally, the disturbance occurs between hours 7 and 8 and consists of simultaneous outage of multiple lines. For the 11hub example, the disturbance takes out three lines, while the larger network experiences a simultaneous outage of four lines.

\section{Simulation RESUlts}

The two systems described in Section $\mathrm{V}$ are simulated according to the flow diagram in Figure 3 for various energy storage scenarios. The energy storage scenarios are considered by varying storage capacity and storage power limits from the nominal values by a given factor. For example, for scenario (capacity, power $)=(0.25,0.10)$, energy storage capacity is at $25 \%$ of nominal and power limits are $10 \%$ of nominal.

We are interested in comparing how different energy storage scenarios affect the average load shed over the 24 hour period. In particular, we will consider the systems with "no" (0), "some" $(\approx 0.25)$, "nominal" $(1.0)$, and "a lot" $(10)$ of storage 
TABLE V

MPC ENERGY SCENARIO RESULTS FOR 11-HUB SYSTEM

\begin{tabular}{ll|cccc}
\hline $\begin{array}{l}\text { Avg. load } \\
\text { shed (\%) }\end{array}$ & & \multicolumn{4}{|c}{ Capacity } \\
& & $\mathbf{0}$ & $\mathbf{0 . 2 5}$ & $\mathbf{1 . 0}$ & $\mathbf{1 0}$ \\
\hline \multirow{2}{*}{ Power } & $\mathbf{0 . 1 0}$ & 12.7 & 10.6 & 9.1 & 8.7 \\
& $\mathbf{1 . 0}$ & 12.7 & 10.8 & 9.0 & 9.2 \\
& $\mathbf{5 . 0}$ & 12.7 & 10.8 & 10.6 & 10.6 \\
\hline
\end{tabular}

TABLE VI

OPEN-LOOP ENERGY SCENARIO RESULTS FOR 11-HUB SYSTEM

\begin{tabular}{ll|cccc}
\hline $\begin{array}{l}\text { Avg. load } \\
\text { shed (\%) }\end{array}$ & & \multicolumn{4}{|c}{ Capacity } \\
& & $\mathbf{0}$ & $\mathbf{0 . 2 5}$ & $\mathbf{1 . 0}$ & $\mathbf{1 0}$ \\
Power & $\mathbf{0 . 1 0}$ & 32.3 & 30.6 & 28.9 & 28.5 \\
& $\mathbf{1 . 0}$ & 32.3 & 32.3 & 30.1 & 29.8 \\
& $\mathbf{5 . 0}$ & 32.3 & 32.3 & 31.9 & 10.4 \\
\hline
\end{tabular}

capacity, while we consider the systems subject to "small" (0.10), "nominal" (1.0), and "large" $(5 \geq)$ power limits.

\section{A. Small System}

In the nominal case, the 11-hub system from Figure 4 undergoes a disturbance that results in the outage of lines 2 , 5 , and 8 . The loss of the three lines leaves line 9 (natural gas) with a significant overload, which must be cleared to avoid tripping the line. Under the MPC scheme, generators are reconfigured (considering power limits), storage is utilized, and minimal load is shed over the 5-minute interval to avoid tripping line 9. The MPODF schedule is updated to reflect the multi-line outage and some load $(\approx 20 \%)$ must be shed until wind-power becomes available towards the end of the day. In the open-loop nominal case, line 9 is not protected and trips after 5 minutes, which leaves the system in a weak state and heavy load-shedding $(\approx 50 \%)$ takes place. For details on the nominal case, we refer the reader to [4].

The results of the 11-hub system are shown in Tables $\mathrm{V}$ and VI. Increasing storage capacity generally reduces the amount of load shed and improves performance of cascade mitigation, which is expected, since more energy can be stored and is available to inject into the system upon the disturbance. However, the general trend suggests that lowering power limits improves performance. The reason behind this trend is that the MPC scheme does not care about the state of the system beyond the halting of the cascade and will maximally utilize (free) stored energy to satisfy demand and avoid shedding load. However, with small power limits, the amount of stored energy is effectively rationed during the cascade mitigation process and some conventional generation is needed to satisfy demand and halt the cascade. This balance between generation and energy utilization allows MPC to halt the cascade and best positions the multi-energy system to satisfy load demand over the subsequent period (following the cascade).

In the open-loop case, the amount of load shed under scenario $(10,5.0)$ is relatively low, because line 9 is "accidentally" not overloaded at minute 5 . The term "accidentally" is used since the open-loop controller attempts to satisfy all demand at the lowest cost with no regard for flow limits. Furthermore, for the $(10,5.0)$ scenario, the open-loop case
TABLE VII

MPC ENERGY SCENARIO RESULTS FOR 69-HUB SYSTEM

\begin{tabular}{ll|cccc}
\hline \multirow{2}{*}{$\begin{array}{l}\text { Avg. load } \\
\text { shed (\%) }\end{array}$} & & \multicolumn{4}{|c}{ Capacity } \\
& & $\mathbf{0}$ & $\mathbf{0 . 2 0}$ & $\mathbf{1 . 0}$ & $\mathbf{1 0}$ \\
\hline & $\mathbf{0 . 1 0}$ & 1.1 & 0.9 & 0.5 & 0.4 \\
Power & $\mathbf{1 . 0}$ & 1.1 & 1.0 & 0.7 & 0.5 \\
& $\mathbf{1 0}$ & 1.1 & 1.0 & 0.7 & 0.7 \\
\hline
\end{tabular}

TABLE VIII

OPEN-LOOP ENERGY SCENARIO RESULTS FOR 69-HUB SYSTEM

\begin{tabular}{ll|cccc}
\hline \multirow{2}{*}{$\begin{array}{l}\text { Avg. load } \\
\text { shed (\%) }\end{array}$} & & \multicolumn{4}{|c}{ Capacity } \\
& & $\mathbf{0}$ & $\mathbf{0 . 2 0}$ & $\mathbf{1 . 0}$ & $\mathbf{1 0}$ \\
\hline \multirow{3}{*}{ Power } & $\mathbf{0 . 1 0}$ & 23.8 & 24.7 & 23.7 & 19.9 \\
& $\mathbf{1 . 0}$ & 23.8 & 23.1 & 23.7 & 14.9 \\
& $\mathbf{1 0}$ & 23.8 & 23.1 & 24.8 & 29.4 \\
\hline
\end{tabular}

sheds marginally less load than the MPC scheme over the 24-hour period. This is because, in our optimization, we do not penalize the spilling of wind power, which means that the MPC scheme can use available energy storage and wind power at the same cost. Thus, stored energy in the future (i.e. beyond the 5-minute prediction window) has no value to the MPC controller. MPC employs available energy storage and spills wind power, while the open-loop case utilizes available wind-power. The MPC response is optimal over the 5-minute period, however with the cascade halted, it leaves the system with less energy stored. This results in a marginal increase in load-shedding over the remaining hours.

\section{B. Large system}

The trends from the 11-hub system apply to the 69-hub system as well, and the results are provided in Tables VII and VIII. As storage capacity increases and power limits decrease, the performance of MPC increases for the same reasons mentioned above.

In the open-loop case, the trend is not as strong, but nonetheless discernible. While the complexity of the network obfuscates the subtleties of the result, it is worth noting that for the scenario without storage (i.e. zero capacity), the amount of load shed is similar to low- and medium-capacity scenarios $(0.2, *)$ and $(1.0, *)$.

The behavior of both the open-loop system and the MPC cascade mitigation scheme are depicted in Figures 5 and 6, for energy scenarios $(0,10),(0.20,10),(1.0,10)$, and $(10,10)$. (Note that thicker lines represent higher capacity scenarios). In fact, for scenarios $(*, 10)$, the open-loop system generally sheds more and more load as storage capacity increases. This is because the open-loop case is uncontrollable in the sense that it does not regard line-flow limits and, for larger systems, this results in significant line tripping as seen in Figure 5(a). At high power limits and with increasing storage capacity, the open-loop controller will inject more stored energy into the system (Figure 5(c)), which, coupled with non-trivial generation levels (Figure 6), results in line tripping (Figure 5(a)) and fragmentation of the system. The fragmentation leads renewables to become isolated from loads, which is evident in the open-loop case in Figure 5(b), since the increase in 
available wind power (in evening and at night) is unable to recover shed load. The fragmentation is most severe in the highest capacity case, where available wind-power is separated from loads and can only be utilized to aimlessly increase energy storage levels.

Note that "No Disturbance" cases represent optimal energy management. However, in the absence of a disturbance, we still need to shed some nominal load when wind-power reaches its nadir and no more power can be injected by conventional generation without exceeding flow limits.

Furthermore, it is worth noticing that the zero-capacity MPC scheme $(0, *)$ sheds only $1.1 \%$ of total load while the optimal MPC storage configuration $(10,0.1)$ sheds $0.4 \%$ of total load. Therefore, one should ask if the investment made in storage devices is worth the marginally improved cascade mitigation performance (a $0.7 \%$ reduction in load shed). Indeed, with an open-loop minimal load shed of $14.9 \%$, the MPC scheme without storage may be a sufficient cascade mitigation solution. However, other factors, such as intermittency and congestion reduction, suggest the need for considering distributed storage devices in the investment process.

\section{CONClusions AND Future Work}

In this paper, we discussed multi-energy systems and a model-predictive cascade mitigation control scheme, within the context of energy hubs. We investigated the performance of the MPC and open-loop schemes under various energy storage scenarios for both small and large multi-energy systems. The results show that sizing and performance ratings of energy storage devices have significant effects on cascade mitigation control in multi-energy systems. Specifically, we conclude that increasing storage capacity improves performance of our MPC scheme, but that power must be carefully regulated. This is because MPC has a tendency to over-exploit stored energy to the detriment of long-term energy requirements.

Further studies will improve upon the line outage model to include probabilistic outage rates determined by cumulative line overloads. In addition, we will couple the MPC scheme and the MPODF schedule to allow for optimal cascade mitigation. Finally, we will include siting of energy storage devices to study the optimal location of energy hubs to reduce network congestion.

\section{REFERENCES}

[1] U.S.-Canada Power System Outage Task Force, "Final report on the August 14, 2003 blackout in the United States and Canada: causes and recommendations," Apr 2004.

[2] M. Geidl and G. Andersson, "Optimal power flow of multiple energy carriers," IEEE Transactions on Power Systems, vol. 22, no. 1, pp. 145 $-155,2007$.

[3] H. Groscurth, T. Bruckner, and R. Kümmel, "Modeling of energyservices supply systems," Energy, vol. 20, pp. 941-958, Jan 1995.

[4] M. Almassalkhi and I. Hiskens, "Cascade mitigation in energy hub networks," IEEE Control and Decision Conference, Dec 2011.

[5] M. Marwali, M. Haili, M. Shahidehpour, and K. Abdul-Rahman, "Short term generation scheduling in photovoltaic-utility grid with battery storage," IEEE Transactions on Power Systems, vol. 13, no. 3, pp. 1057 $-1062,1998$.

[6] J. P. Barton and D. G. Infield, "Energy storage and its use with intermittent renewable energy," IEEE Transactions on Energy Conversion, vol. 19, no. 2, 2004.

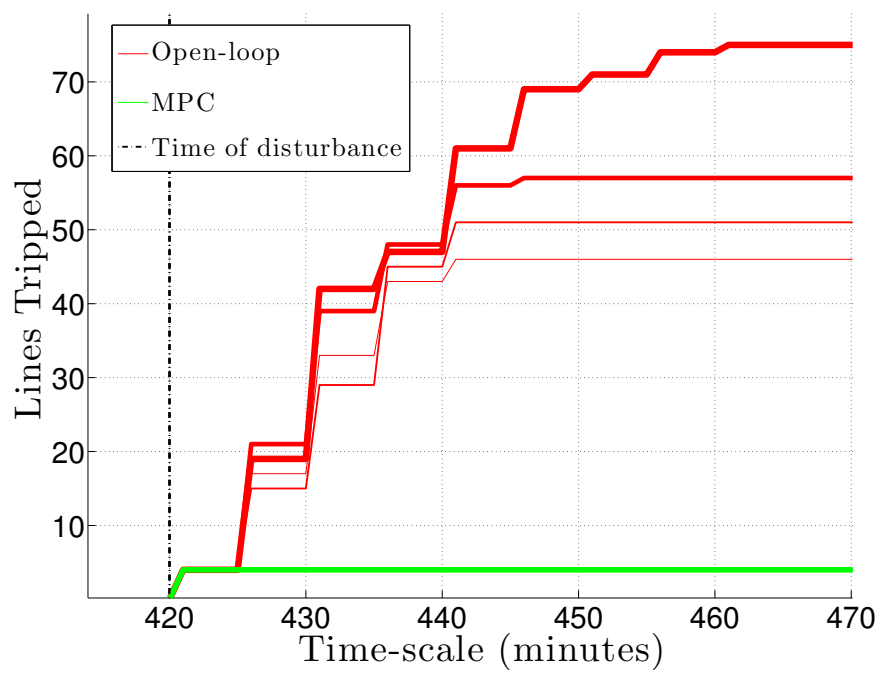

(a) Lines tripped on fast time-scale

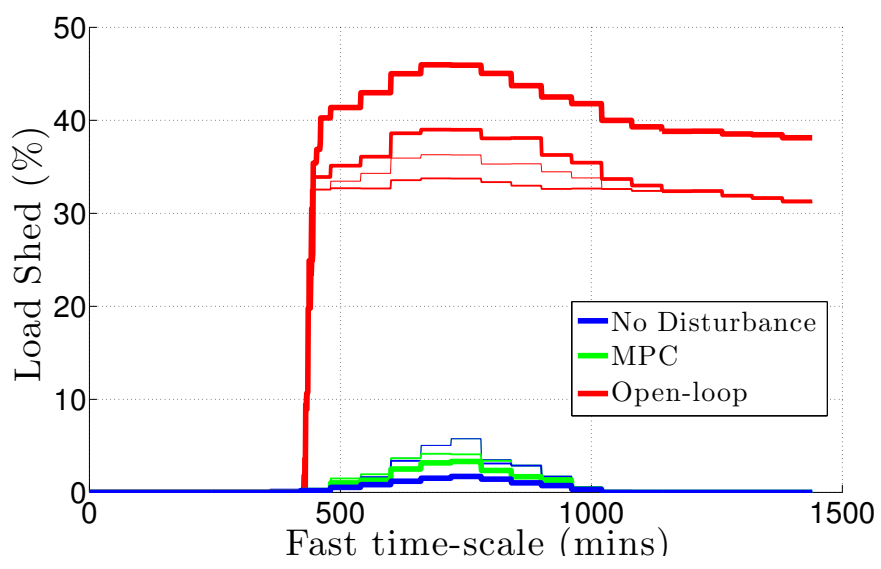

(b) Load shedding on fast time-scale

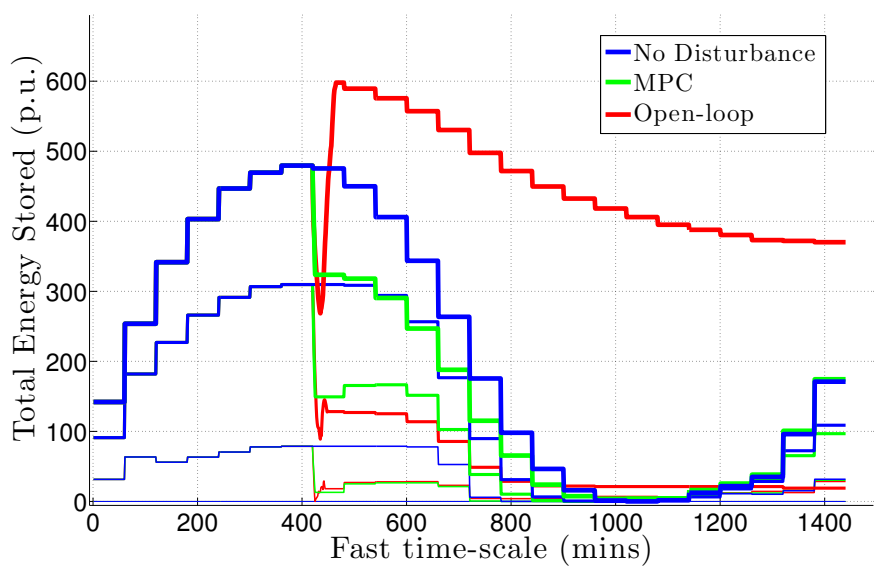

(c) Energy storage management on fast time-scale

Fig. 5. Cascade simulation results from large 69-hub system with power limits at 10 times nominal. As storage capacity increases, the open-loop case undergoes increasingly significant cascading. (Note: thicker lines $\Rightarrow$ higher capacity). 


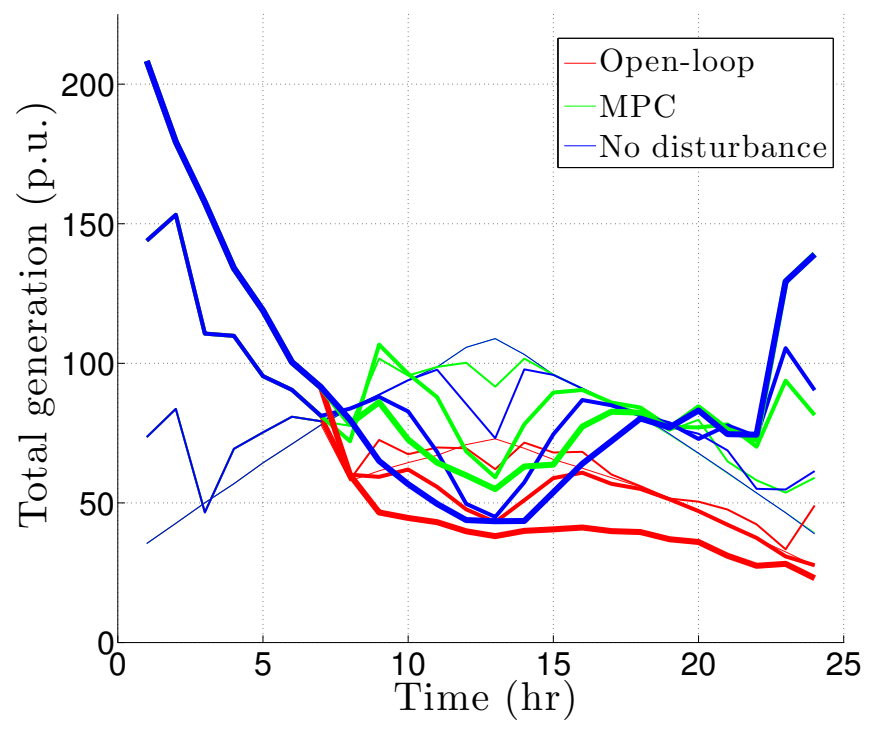

Fig. 6. Generator levels for energy scenarios with ten times nominal power limits. As storage capacity increases, more off-peak power is utilized for storage, shaving off peaks in generation. (Note: thicker lines $\Rightarrow$ higher capacity).

[7] H. Bludszuweit and J. Dominguez-Navarro, "A probabilistic method for energy storage sizing based on wind power forecast uncertainty," IEEE Transactions on Power Systems, vol. 26, no. 3, pp. 1651 - 1658, 2011.

[8] S. Buldyrev, R. Parshani, G. Paul, H. E. Stanley, and S. Havlin, "Catastrophic cascade of failures in interdependent networks," Nature, vol. 464, pp. 1025-1028, Apr 2010.

[9] M. Shahidehpour, Y. Fu, and T. Wiedman, "Impact of natural gas infrastructure on electric power systems," Proceedings of the IEEE, vol. 93, no. 5, pp. 1042 - 1056, 2005.

[10] C. Unsihuay-Vila, J. Marangon-Lima, A. de Souza, I. Perez-Arriaga, and P. Balestrassi, "A model to long-term, multiarea, multistage, and integrated expansion planning of electricity and natural gas systems," IEEE Transactions on Power Systems, vol. 25, no. 2, pp. $1154-1168$, 2010.

[11] M. Almassalkhi and I. Hiskens, "Optimization framework for the analysis of large-scale networks of energy hubs," Power Systems Computation Conference, Aug 2011.
[12] D. Bienstock and A. Verma, "The N-k problem in power grids: new models, formulations, and numerical experiments," SIAM Journal on Optimization, vol. 20, p. 2352, 2010.

[13] B. Carreras, V. Lynch, M. Sachtjen, and I. Dobson, "Modeling blackout dynamics in power transmission networks with simple structure," 36th Hawaii International Conference on System Sciences, Jan 2001.

[14] L. Bahiense, C. Oliveira, M. Pereira, and S. Granville, "A mixed integer disjunctive model for transmission network expansion," IEEE Transactions on Power Systems, vol. 16, no. 3, pp. 560-565, 2001.

[15] B. Joseph and F. W. Hanratty, "Predictive control of quality in a batch manufacturing process using artificial neural network models," Ind. Eng. Chem. Res., vol. 32, pp. 1951-1961, 1993.

[16] F. Metzger (of Kinder-Morgan). Personal communication, November 4th 2011.

[17] Z. Wang, R. Thomas, and A. Scaglione, "Generating random topology power grids," Proceedings of the 41st Hawaii International Conference on System Sciences, pp. 1-9, 2008.

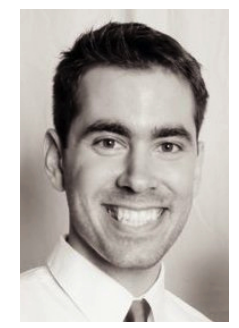

Mads R. Almassalkhi (S`06) was born in Copenhagen, Denmark. He received a B.S. degree in electrical engineering with a dual major in applied mathematics from the University of Cincinnati, Cincinnati, USA, in 2008, and an M.S. in electrical engineering: systems from the University of Michigan, Ann Arbor, USA, in 2010, where he is currently pursuing his Ph.D degree. His research is dedicated to modeling, optimization, and control of multiple energy carrier systems and fleets of plug-in electric vehicles.

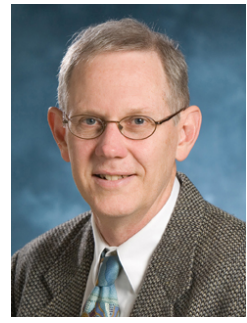

Ian A. Hiskens (S‘77-M'80-SM'96-F`06) received the B.Eng. degree in electrical engineering and the B.App.Sc. degree in mathematics from the Capricornia Institute of Advanced Education, Rockhampton, Australia, in 1980 and 1983, respectively, and the $\mathrm{Ph} . \mathrm{D}$. degree in electrical engineering from the University of Newcastle, Australia, in 1991.

$\mathrm{He}$ is the Vennema Professor of Engineering in the Department of Electrical Engineering and Computer Science, University of Michigan, Ann Arbor. He has held prior appointments in the Queensland electricity supply industry, and various universities in Australia and the United States. His major research interests lie in the area of power system analysis, in particular system dynamics and control, and security assessment. His recent activity has focused largely on integration of new forms of generation and load. Other research interests include nonlinear and hybrid dynamical systems.

Dr. Hiskens is actively involved in various IEEE societies, and is VP of Finance of the IEEE Systems Council. He is a Fellow of Engineers Australia and a Chartered Professional Engineer in Australia. 